\title{
Chemische Wirkstoffe im Nestbau-, Sexual- und Kampfverhalten des Sonnenbarsches Lepomis gibbosus (L.) (Centrarchidae, Teleostei) ${ }^{1}$ )
}

\author{
Von Bernd Kramer ${ }^{2}$ )
}

Mit 10 Abbildungen

Eingegangen am 14. 4. 1972

\section{A. Einleitung}

Eine Analyse des Fortpflanzungs- und Kampfverhaltens des Sonnenbarsches und orientierende Versuche zur Frage der Steuerung durch Sexualhormone (KramER 1969, 1971; KRAMER, Molenda und FIEDler 1969) sind die Grundlagen für diese Arbeit. In ihr wird der Einfluß der langfristig wirkenden humoralen Faktoren weiter untersucht und außerdem der Frage nach den Ursachen für momentane Stimmungsänderungen nachgegangen.

Es hatte sich gezeigt, daß das Antitestosteron Cyproteronacetat (ScHERING) das Nestbauverhalten von Sonnenbarsch- $\hat{~} \hat{~} \hat{\delta}$ vollständig zu unterdrücken vermag (KRAMER 1969, 1971). Im Einklang hiermit steht der Befund, daß von $7 \mathrm{mit}$ Testosteron $(0,03 \mathrm{mg} / \mathrm{g} \mathrm{KG})$ injizierten Tieren ( 4 davon 9 우), die vorher nicht gebalzt oder Nester gebaut hatten, alle nach 3 Tagen riesige Gruben ausschlugen. Ein Bericht von SMITH (1969) läßt ebenfalls auf eine Steuerung durch Androgen schließen: kastrierte Tiere konnten erst nach Methyltestosteron-Gaben wieder Nester bauen.

Auf die Aggressivität hatte Cyproteronacetat eine nur mäßig hemmende Wirkung, in stärkerem Maß wurde sie schon am 1. Tag p.I. durch gelöstes, schnell resorbierbares Methyltestosteron vermindert. Tiere in der Bau-Balz-Laichphase verfügen, wie an ihrer Baustimmung abzulesen ist, selber schon über einen hohen Androgenspiegel. Exogenes Testosteron verstärkte offenbar die Hemmwirkung körpereigenen Androgens auf die Gonadotropin-Ausschüttung, die über einen negativen Rüdkkopplungsmechanismus ausgeübt wird, bis zu einer vollkommenen Blodkade. Dies mußte folglich die Testosteron-Synthese im Hoden verringern. Nach einer nochmaligen Testosteron-Injektion, die 3 Wochen später erfolgte, stieg die Aggressivität der Tiere wieder an; diese Befunde ließen sich durch ein synergistisches Zusammenwirken von Testosteron und Gonadotropin für die Motivierung aggressiven Verhaltens erklären. Die Existenz eines Rückkopplungsmechanismus zwischen Hypophyse und Gonade (Endprodukthemmung) auch bei Teleosteern, wie er hierfür vorausgesetzt wird, ist durch eine Reihe von histologischen Untersuchungen an hormonbehandelten Fischen (auch Sonnenbarschen) wahrscheinlichgemacht (kurze Übersicht s. KRAMER 1971).

1) Dissertation der naturwissenschaf tlichen Fakultät der Universität Frankfurt/Main.

2) Herrn Prof. Dr. M. Lindauer danke ich sehr für die kritische Durchsicht des Manuskriptes und wertvolle Ratschläge. 
SMIth (1970) berichtet, daß die Wassertemperatur und nicht Androgen oder injiziertes menschliches Choriongonadotropin die Aggressivität von Sonnenbarschen beeinflusse. Das Ergebnis mit Androgen wird in dieser Arbeit widerlegt, der Aussage über die Wirkung von Gonadotropin muß in der Diskussion anhand eigener Versuche mit Luteinisierendem Hormon aus Säugetier-Hypophysen widersprochen werden.

Sexualverhalten konnte nicht durch Cyproteronacetat, sondern nur durch gelöstes Testosteron unterdrückt werden (KRAMER 1969, 1971); dies legte eine (wenigstens überwiegende) Steuerung durch Gonadotropin nahe.

Für die hormonale Kontrolle des aggressiven und des Sexualverhaltens lagen somit bisher lediglich indirekte Belege vor; ein Ziel der vorliegenden Arbeit ist es, den direkten Beweis zu erbringen.

Die oben angedeutete zweite Frage, die in dieser Arbeit untersucht wird, war die nach der Ursache für die immer wieder am Verhalten zu beobachtenden momentanen Stimmungsänderungen. Neuere biochemische, elektrophysiologische und pharmakologische Untersuchungen (s.

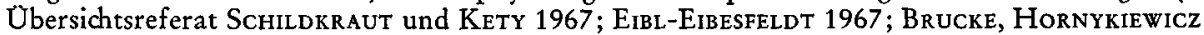
und Sigg 1969; Kuschinsky und Lüllmann 1970) sprechen dafür, daß dem Catecholaminstoffwechsel im Gehirn hierbei eine zentrale Bedeutung zukommt. Mit Hilfe der Psychopharmaka Reserpin und Chlorpromazin, die die Ausschüttung der biogenen Amine in unterschiedlicher Weise beeinflussen, war eine Klärung dieser Frage zu erhoffen.

Diese Arbeit wurde im ehemaligen Zoologischen Institut der Universität Frankfurt am Main durchgeführt. Ich danke Herrn Prof. Dr. M. Lindauer und Herrn Prof. Dr. D. BurkHARDT für die Uberlassung eines Arbeitsplatzes und für großzügige Unterstützung. Meinem verehrten Lehrer, Herrn Prof. Dr. K. FiEdLer, gilt mein besonderer Dank.

Das National Institute of Health, Endocrinology Study Section, Bethesda (Md.), USA, stellte dankenswerterweise die hochgereinigten Hormone NIH-LH-S14 und NIH-LH-B4 zur Verfügung. Der Firma Imperial Chemical Industries (ICI), Ltd., Macclesfield, England, sowie der Firma Bayer, Leverkusen, danke ich für die kostenlose Überlassung von Methallibur bzw. Megaphen.

Zu Dank verpflichtet bin ich auch der Studienstiftung des Deutschen Volkes, die mich finanziell unterstïtzte.

\section{B. Material und Methoden}

Die Ergebnisse wurden an Sonnenbarsch- $\delta \hat{\delta}$ gewonnen, die aus oberbayerischen Seen gefangen worden waren. Sie hatten ein Gewicht von $16-63 \mathrm{~g}$ und eine Standardlänge von $8-12 \mathrm{~cm}$. Die Tiere wurden bis zur Verwendung im Experiment in 260-1-Becken unter Langtagsbedingungen (16 Std. Licht, $8 \mathrm{Std}$. Dunkelheit) gehalten. Im Versuch waren je 2 Tiere voneinander isoliert in 60-1-Becken untergebracht, die eine undurchsichtige Plastik-'Trennscheibe unterteilte.

Die Stärke der aggressiven und der Sexualtendenz eines ơ ließ sich nach FiEdLer (1964, 1967) quantitativ messen, indem ich ihm täglich für $5 \mathrm{Min}$. ein $\oint$ dazusetzte. Dies führte bei den meisten $\hat{\partial} \hat{\partial}$ zum Ansteigen der Aggressivität und zur Auslösung des Nestbau- und Sexualverhaltens im Verlauf von 1-2 Wochen. Innerhalb dieses Zeitraumes könnte man die Wirkung eines injizierten Hormons nicht sicher vom trophischen Effekt (HeILIGenberg 1963) unterscheiden, den das tägliche, fünfminütige Anbieten eines $O$ hat. Daher wartete ich konstante Meßwerte $a b$, was zugleich als Kontrolle dafür gilt, daß bei dem jeweiligen Tier entsprechendes Verhalten auszulösen ist. Dann versuchte ich, die Tiere mit Hilfe eines Gonadotropinhemmstoffes (Methallibur) wieder in die neutrale Phase zu versetzen. Jetzt konnte erwartet werden, $\mathrm{da}$ die injizierten Hormone die gleiche Wirkung wie homologe fischeigene beim Ubergang von der neutralen zur Bau-Balz-Laichphase hätten.

Methallibur hat offenbar eine leichte Nebenwirkung auf die Schilddrüse (HoAR et al. 1967; WIEBE 1968 und 1969; Walpole 1965; Tulloch et al. 1963). Nicht durch Methallibur beeinflußt wird die Funktion der Nebennierenrinde (Walpole 1965).

In dieser Arbeit ist die Kampfstärke ${ }^{1}$ ) in 2 Werten wiedergegeben: der größere ist der Spitzenwert („maximale Kampfstärke“), der während einer der fünf Kampfminuten erreicht wurde, der kleinere der Durchschnittswert der 5 einzeln registrierten Minuten („durchschnittliche Kampfstärke“). Das Maß für die Sexualtendenz ist die Zahl der Kreise, die der Versuchsfisch in der Nestgrube mit dem $O$ (oder falls es stehenblieb, um es herum) ausführte. Die Intensität des Nestbauens („Fegen“ des Kiesbodens mit der Schwanzflosse) wurde vor und nach jedem Kampfversuch in einer jeweils 15 minütigen Sitzung ermittelt, indem die Dauer

1) = Zahl der Stöße (mit 1 bewertet) plus Stoßintentionen (mit $1 / 2$ bewertet) pro Minute (FiedLeR 1967). 
jeder einzelnen Nestbauhandlung mit der Stoppuhr gemessen und der Durchschnittswert für eine Minute errechnet wurde. Auf die Angabe der Häufigkeit der Nestbauhandlungen pro Minute konnte verzichtet werden, da ihr Kurvenverlauf praktisch identisch ist mit dem Kurvenverlauf für die Dauer dieser Handlungen (KRAMER 1971; hier ist auch eine Beschreibung der gemessenen Verhaltensweisen und eine Analyse ihrer Beziehungen untereinander gegeben).

Der Gonadotropinhemmstoff Methallibur2) (I.C.I. 33828), ein Bis-Thioharnstoffderivat, wurde in der berechneten Menge in eine $10 \mathrm{Vol}$ \% ige Lösung von 1,2-Propylenglykol in aqua dest. eingebracht und mit einem POTTER-ElVEHJEM-Homogenisator mikrokristallin zerteilt. Die perorale Infusion erfolgte mit Hilfe eines dünnen, auf eine Kanüle Nr.16 aufgestedkten Plastik-Schlauches, dessen freies Ende vorsichtig bis in den Magen des narkotisierten Tieres vorgeschoben wurde (die hierfür erforderliche Länge war zuvor an toten Fischen ermittelt worden). Für die intramuskuläre Injektion verwendete ich die feinere Kanüle Nr. 20 (weitere Angaben s. KRAMER 1971).

HoAr et al. (1967) berichten von einer erhöhten Mortalität nach intramuskulären Methallibur-Injektionen beim Stichling (50\%), aber nicht beim Goldfisch und Cymatogaster; zum Teil ist sie auf den der Suspension zugesetzten Stabilisator ("Tween 80“) zurückzuführen. Die überlebenden Tiere waren in offensichtlich gutem Gesundheitszustand. WIEBE $(1968,1969)$ gab seinen Tieren Methallibur als Suspension ins Aquarienwasser, sie überlebten im allgemeinen zu $100 \%$ und waren nicht geschädigt. Auch GALOw (1969), der Methallibur seinen Tieren (Betta splendens) ebenfalls suspendiert im Aquarienwasser und außerdem peroral verabreichte, berichtet, daß er keinerlei toxische Erscheinungen erkennen konnte. Da GALOW mit im Wasser suspendiertem Methallibur keine Hemmung des Schaumnestbaus erzielen konnte, und die verschiedensten Detergentien diese Substanz nicht in Suspension erhielten, gab ich es meinen Tieren peroral und intramuskulär. Störend machte sich eine erhöhte Anfälligkeit der Tiere gegenüber Ichthyophthirius bemerkbar, bei intramuskulär injizierten (besonders nach mehrmaliger Injektion) konnte es auch Entzündungen geben, die meistens tödlich verliefen. $\mathrm{Da}$ die Substanz vom Hersteller nicht steril abgepadkt und wegen ihrer Temperaturempfindlichkeit (hoher Stickstoffgehalt) nicht autoklaviert werden konnte, war es nicht möglich, die Zahl der Erkrankungen, die $40 \%$ betrug, zu verringern. Bei den gesund gebliebenen Tieren, auf die sich die Ergebnisse dieser Arbeit beziehen, konnte ich jedoch keinerlei pathologische Veränderungen im Aussehen der Tiere oder ihrem Verhalten erkennen.

Die Tiere erhielten Reserpin in Form des Präparates „Sedaraupin“ der Fa. Boehringer Mannheim G.m.b.H. (Charge 63445) intramuskulär injiziert.

Chlorpromazin wurde als „Megaphen" (Fa. Bayer, Charge 7B) dem Aquarienwasser zugefügt. Da sich diese Substanz im Licht allmählich zersetzt, war es angezeigt, wie RENSCH und DüCKER (1966), die dieses Psychopharmakon in einem Lernexperiment mit Goldfischen benutzt hatten, am 1. Tag dem Aquarienwasser die volle Konzentration zuzufügen und an den folgenden Tagen die Hälfte des Wassers durch frisches zu ersetzen und die fehlende Chlorpromazinmenge zu ergänzen. Die genaue Konzentration ist, wie RENSCH und DüCKER (1966) ausführten, nicht angebbar, da ein Teil über die Mundhöhlenschleimhaut und die Kiemen resorbiert, ein anderer vom Licht allmählich zersetzt wird. Nach 6 Behandlungstagen zeigten die Tiere erstmals abnorme Körperhaltungen bei gleichzeitigem Anschwellen der Körperflanken (s. auch RENSCH und DüCKER 1966). Chlorpromazin wirkt in höherer Konzentration oder über längere Zeit schädigend auf die Leber (Abbau des Chlorpromazins in der Leber s. WIRTH et al. 1958; KuschinsKy und LüLLMANN 1970). Der Aussage über die Verhaltenswirksamkeit des Chlorpromazins sollen daher nur die Werte für die ersten 3 Tage zugrunde gelegt werden (die Werte für die folgenden 14 Tage sind bei Kramer Diss. 1971 einzusehen).

Testosteron war synthetisches $17 \alpha$-Methyltestosteron (Charge 422770) der Fa. Merdk. Das verwendete Gonadotropin (NIH-LH-S14 und NIH-LH-B4) ist aus Säugerhypophysen isoliertes Luteinisierendes Hormon (National Institute of Health, USA).

Die Gonaden der Versuchs- und Kontrolltiere fixierte ich nach beendetem Versudh in Bouinscher Lösung. Die $7 \mu$ dicken Schnitte wurden nach der GoldNERschen Trichrom-Methode mit oder ohne vorherige Alldehydfuchsinbehandlung (GoMORI-GABE) gefärbt (s. RomeIs 1968).

Die statistische Bearbeitung der Meßwerte erfolgte mit Hilfe des t-Testes für die Differenz $z$ weier Mittelwerte aus verbundenen Stichproben. Hierbei ist die Testgröße T definiert als

$$
\mathrm{T}=\frac{\overline{\mathrm{d}}}{\mathrm{s}_{\mathrm{d}}} \sqrt{\mathrm{N}}
$$

wobei $\bar{d}$ die Differenz der Mittelwerte der beiden Meßreihen, $s_{d}$ die Standardabweichung der Differenz dieser Reihen und N die Zahl der Individuen ist (s. z. B. Cavalli-Sforza 1969).

2) 1- $\alpha$-Methylallylthiocarbamoyl-2-Methylthiocarbamoyl-Hydrazin. 


\section{Ergebnisse}

I. Der Einfluß von Methallibur, von Hormonen nach Methallibur-Vorbehandlung und von Psychopharmaka auf das Verhalten

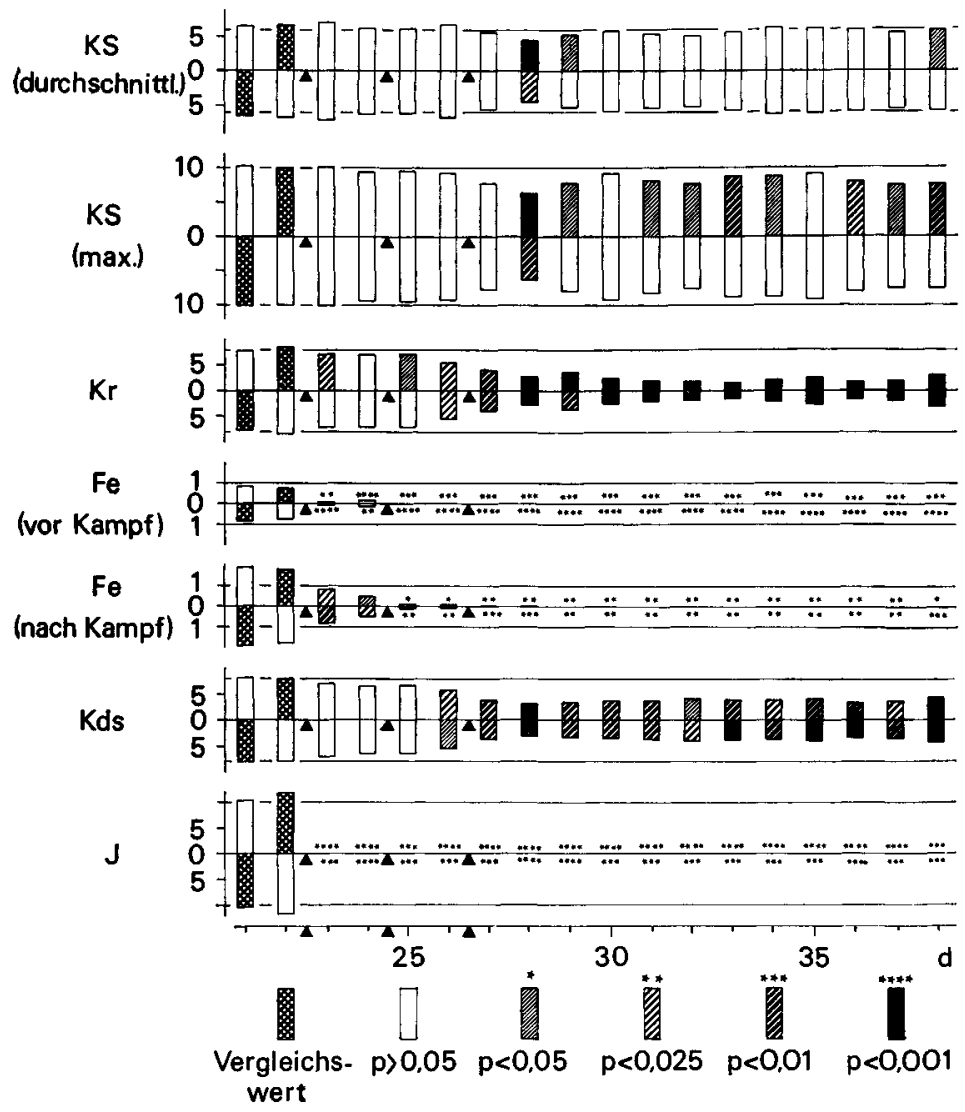

Abb. 1: Die Wirkung von drei Methallibur-Injektionen $(0,2 \mathrm{mg} / \mathrm{g} \mathrm{KG})$, die einander im $\mathrm{Ab}-$ stand von 2 Tagen folgten, auf die Kampfstärke (KS), das Kreisen (Kr), das Fegen (Fe), das Kiemendedkelspreizen (Kds) und das Jagen (J) von Sonnenbarsch- $\delta \hat{\delta} \delta(n=9$; Juni 1970). Abszisse: Zeit in Tagen; Ordinaten: Anzahl der Handlungen/Min. (jedoch bei Fegen: Dauer der Nestbauhandlungen in sec/Min.). Die Injektionen erfolgten unmittelbar nad den Verhaltensmessungen an den Tagen 22, 24 und 26 (s. Pfeile). Die nach oben aufgetragenen Meßwerte wurden daraufhin überprüft, ob sie vom Meßwert für den Tag der Injektion (Tag 22, kreuzschraffiert) verschieden sind (Bedeutung der verschiedenen Schraffuren oder Anzahl von Sternchen s. untere Zeile); nach unten aufgetragen ist eine ebensolche Analyse, bei der als Vergleichsbasis der Vortag der Injektion benutzt wurde

\section{Die Wirkung von Methallibur}

Abb. 1 (Juni 1970) zeigt die Wirkung von intramuskulär injiziertem Methallibur ( $3 \times 0,2 \mathrm{mg} / \mathrm{g} \mathrm{KG}$ in 2tägigem Abstand) auf das Sexual-, Nestbauund Kampfverhalten sowie auf 2 Verhaltensweisen des Konfliktes zwischen aggressiver und Sexualtendenz (KRAMER 1971), das Kiemendeckelspreizen und Jagen. $\mathrm{Ab}$ dem 5. Behandlungstag ist die Sexualtendenz (Kreisen) signifikant vermindert gegenuiber 2 Vergleichstagen vor der Behandlung. Nestbauen 
(Fegen) vor dem Kampf war ab dem 3. Behandlungstag vollständig gehemmt (Unterschied gesichert), Fegen nach dem Kampf konnte $\mathrm{ab}$ dem 7. Behandlungstag nicht mehr registriert werden (Unterschied mit $\mathrm{p}<0,025$ auffällig). Bei der durchschnittlichen Kampfstärke liegt nur am 6. Behandlungstag ein beachtenswerter Abfall gegenüber beiden Vergleichstagen vor; die maximale Kampfstärke ist gesichert vermindert an diesem Tag und an einer Reihe von weiteren Tagen auffällig vermindert gegenüber einem der beiden Vergleichstage. Kiemendeckelspreizen tritt ab dem 5 . Behandlungstag gesichert weniger häufig auf (bei 4 Werten ist der Unterschied jedoch nur auffällig: 0,05>p $>0,01$ ). Jagen ist ab dem 1. Tag nach Behandlungsbeginn fast vollständig gehemmt; alle Werte sind signifikant geringer als beide Ausgangswerte.

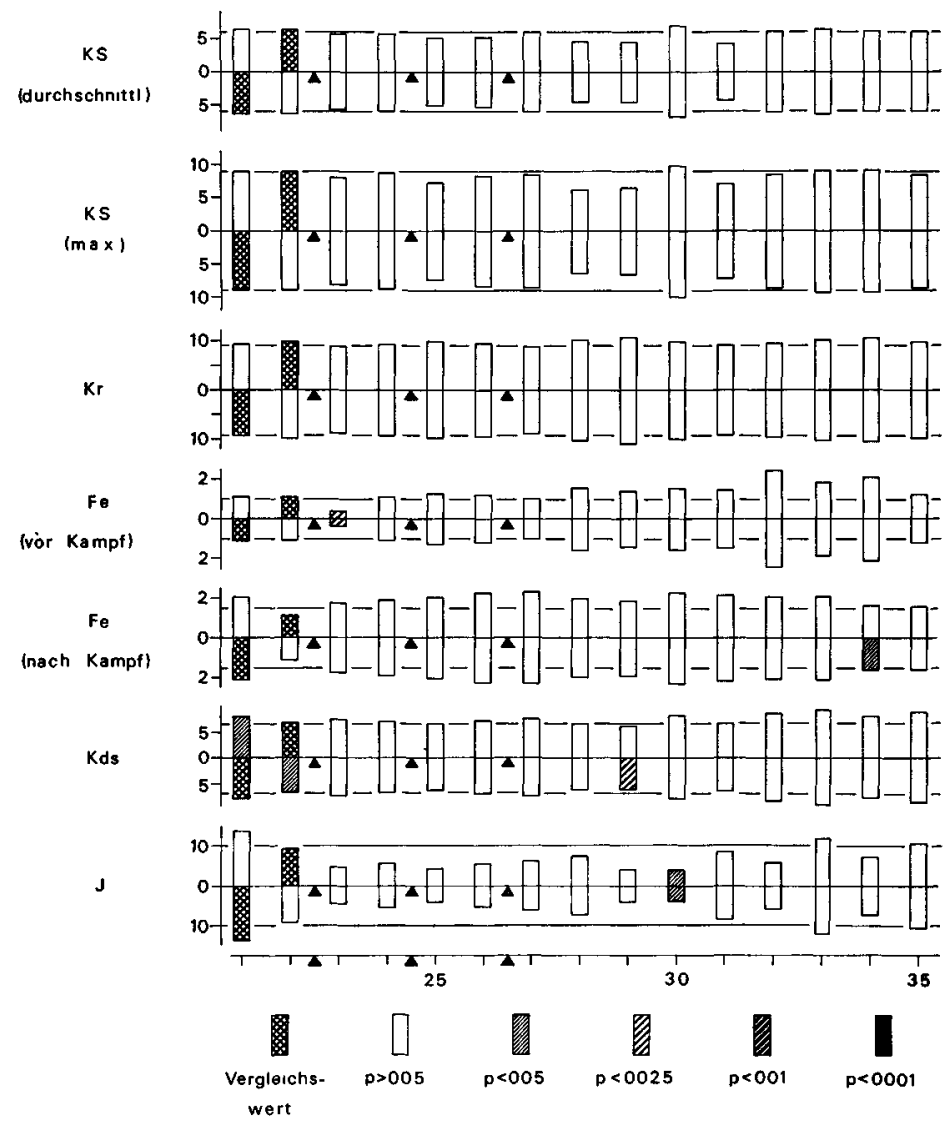

Abb. 2: Die Wirkung dreier Injektionen einer 10 Vol.\%igen wäßrigen Propylenglykol-Lösung, die einander im Abstand von 2 Tagen folgten, auf das Verhalten von Sonnenbarsch- $\delta \hat{\delta}$ $(n=5)$. Injizierte Volumina gleichen den in Abb. 1 verwendeten. Sonst wie Abb. 1

Bei den zur gleichen Zeit behandelten Kontrolltieren (Abb. 2) traten nach den Injektionen keine signifikanten Unterschiede gegenüber den Ausgangswerten auf; lediglich beim Fegen, Kiemendeckelspreizen und Jagen wurde je einmal eine beachtenswerte Verminderung registriert. Die Wirkung der Kontrollbehandlung verglichen mit derjenigen der Methallibur-Behandlung ist somit sehr gering. 
Tab.1: Die Wirkung von täglich peroral verabreichtem Methallibur auf das Verhalten von Sonnenbarsch- $\hat{\partial} \hat{\partial}$. Es wurde je ein Mittelwert aus 5 Tagen vor Behandlungsbeginn (Ausgangswert) und 5 Tagen vor Behandlungsende gebildet; die prozentuale Abweichung vom Ausgangswert ist in der Tabelle angegeben (z.B.,$-100^{\prime \prime}=$ vollständige Hemmung). Bei Gruppe A $(0,1 \mathrm{mg} / \mathrm{g} \mathrm{KG)}$ erstreckte sich die Behandlungsdauer über 13 Tage, bei Gruppe B $(0,2 \mathrm{mg} / \mathrm{g} \mathrm{KG})$ und der Kontrollgruppe über 20 Tage. Die Kontrolltiere erhielten eine 10 Vol.-\%ige wäßrige Propylenglykol-Lösung; die infundierten Volumina gleichen denen der Gruppe $B$ verabreichten. $p$-Werte $>0,05$ sind nicht angegeben

\begin{tabular}{|l|c|c|c|}
\hline Versuchsgruppe & $\begin{array}{c}A \\
(n=4)\end{array}$ & $\begin{array}{c}\text { B } \\
(n=9)\end{array}$ & $\begin{array}{c}\text { Kontrolle } \\
(n=10)\end{array}$ \\
\hline Kreisen & $\begin{array}{c}-90 \\
(p<0,01)\end{array}$ & $\begin{array}{c}-71 \\
(p<0,001)\end{array}$ & \pm 0 \\
\hline $\begin{array}{l}\text { Kampfstärke } \\
\text { (durchschnittl.) }\end{array}$ & $\begin{array}{c}-69 \\
(p<0,01)\end{array}$ & +21 & +2 \\
\hline $\begin{array}{l}\text { Kampfstärke } \\
\text { (max.) }\end{array}$ & $\begin{array}{c}-65 \\
(p<0,01)\end{array}$ & +7 & +8 \\
\hline Kiemendeckelspreizen & $\begin{array}{l}-91 \\
(p<0,001)\end{array}$ & $\begin{array}{c}-44 \\
(p<0,01)\end{array}$ & -17 \\
\hline Fegen nach dem Kampf & $\begin{array}{c}-100 \\
(p<0,001)\end{array}$ & $\begin{array}{c}-100 \\
(p<0,025)\end{array}$ & -14 \\
\hline Fegen vor dem Kampf & $\begin{array}{c}-100 \\
(p<0,025)\end{array}$ & $\begin{array}{c}-100 \\
(p<0,001)\end{array}$ \\
\hline Jagen & $\begin{array}{c}-100 \\
(p<0,05)\end{array}$ & $\begin{array}{c}-100 \\
(p<0,01)\end{array}$ \\
\hline
\end{tabular}

Tab. 1 faßt die Wirkung von peroral verabreichtem, suspendiertem Methallibur auf 2 Versuchsgruppen zusammen; einer in der Tabelle aufgeführten Kontrollgruppe wurde nur die Suspensionsflüssigkeit gegeben. Auch hier hatte $\mathrm{Me}-$ thallibur eine stark hemmende Wirkung auf das Sexualverhalten (Unterschiede signifikant), während die Werte der Kontrollgruppe unverändert blieben. Für Kiemendeckelspreizen und Fegen nach dem Kampf sind die Verhältnisse ähnlich, während beim Fegen vor dem Kampf und Jagen auch bei den Kontrolltieren eine gesicherte (wenn auch weniger starke) Hemmung registriert wurde. Nur die Tiere der Gruppe A zeigten nach der Methallibur-Behandlung eine gesichert geringere Aggressivität (Abnahme um rund $70 \%$ ), während die Tiere der Gruppe B aggressiver wurden (Anstieg um rund 20\%, ungesichert). Die Gruppe A wurde im November untersucht, die Gruppe B im April; für beide Gruppen galten die gleichen Temperatur- und Lichtbedingungen. Die Erklärung dafür, daß sich die Kampfstärke bei Gruppe B ebenso wie bei der oben abgehandelten intramuskulär injizierten Gruppe (Juni) nicht oder nicht dauerhaft hemmen ließ, ist daher wahrscheinlich in einer jahreszeitlich veränderlichen, endogen verursachten Schwankung der Hormonsekretion zu suchen.

Beginnend vom 4. bis 5. Behandlungstag an zeigten alle mit Methallibur behandelten Tiere für weitere 4-5 Tage eine Brutpflegeverhaltensweise, das Fächeln (Beschreibung siehe Kramer 1971).

\section{Die Wirkung von exogenem Testosteron auf mit Methallibur gehemmte Tiere}

$\mathrm{Da}$ Methallibur die Gonadotropin-Ausschüttung und/oder -Synthese hemmt, Gonadotropin aber für die Synthese des Gonadenhormons Testosteron benötigt wird, sollten mit Methallibur vorbehandelte Tiere einen niedrigen Androgen-Spiegel aufweisen. Die Injektion von Testosteron müßte dann zeigen, welche Verhaltensweisen androgenabhängig sind. 


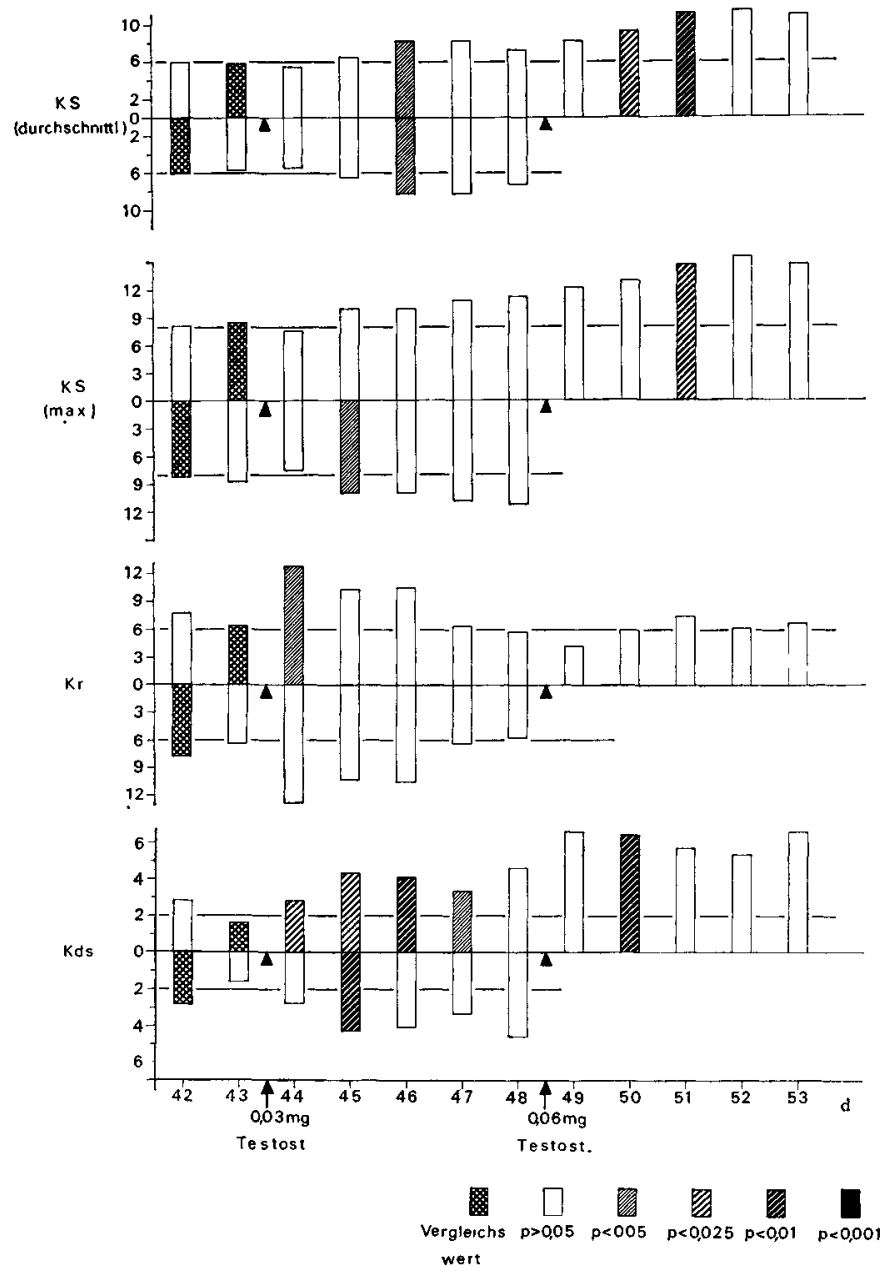

Abb.3: Die Wirkung von injiziertem Testosteron auf das Verhalten von Sonnenbarsch- $\hat{o} \hat{o}$, die mit Methallibur vorbehandelt worden waren $(n=5$, ab Tag 48: $n=4$; Juli 1970). Die Injektionen erfolgten unmittelbar nach den Verhaltensmessungen an den Tagen 43 und 48. Die Mengenangaben verstehen sich pro Gramm Körpergewicht. Vergleichswert für die Tage $49-53$ ist Tag 48 . Sonst wie Abb. 1

Fünf gut balzende $\hat{\delta} \hat{\delta}$ erhielten in 2tägigem Abstand $3 \times 0,2 \mathrm{mg} / \mathrm{g} \mathrm{KG}$ Methallibur intramuskulär injiziert; am 16. Tag nach der 1. Injektion bekamen die Tiere noch einmal eine hohe Dosis $(0,4 \mathrm{mg} / \mathrm{g} \mathrm{KG})$ zur Sicherstellung einer längerfristigen Hemmung („Depoteffekt": unter der Haut konnte man die injizierte Substanz erkennen und ihr langsames Verschwinden innerhalb von 14 Tagen bis 3 Wochen verfolgen). Vier Tage nach der letzten Methalliburinjektion $^{3}$ ) erhielten die Tiere $0,03 \mathrm{mg} / \mathrm{g}$ KG Testosteron (Abb. 3). Drei Tage später stieg die Kampfstärke um durchschnittlich $44 \%$ an $(\mathrm{p}<0,05)$. Am 1. Tag vermehrte sich die Sexualtendenz um das Doppelte $(p<0,05)$, jedoch liegen die Werte für den 2 bis 5 . Tag niedriger, und der Unterschied ist nicht beachtens-

3) Hemmung der Sexualtendenz gegenüber dem Ausgangswert: $85 \%(\mathrm{p}<0,001)$; des Kiemendeckelspreizens: $79 \%(\mathrm{p}<0,01)$; der durchschnittlichen und maximalen Kampfstärke: (je $14 \%$ ungesichert); Fegen und Jagen waren zu $100 \%$ gehemmt. 
wert $(p>0,05)$. Kiemendeckelspreizen erhöhte sich an den ersten 4 Tagen um $75 \%(\mathrm{p}<0,025), 169 \%(\mathrm{p}<0,025), 156 \%(\mathrm{p}<0,01)$ und $110 \%(\mathrm{p}<0,05)$. Vergleicht man die nach der Testosteron-Injektion gemessenen Werte auch mit dem Wert für den Vortag dieser Injektion, so findet man für die Kampfstärke ebenfalls einen beachtenswerten und für Kiemendeckelspreizen einen signifikanten Unterschied, nicht aber für die Sexualtendenz.

Fünf Tage später erhielten die Tiere Testosteron in der doppelten Dosis $(0,06 \mathrm{mg} / \mathrm{g} \mathrm{KG})$. Wiederum stieg die durchschnittliche Kampfstärke an: die mittlere Zunahme betrug am 2. und 3. Tag nach dieser Injektion 27 und 55\% $(p<0,025$ und $p<0,01)$. Der Anstieg der maximalen Kampfstärke am 3. Tag um $30 \%(p<0,025)$ ist auffällig. Die Werte für die Sexualtendenz an den Tagen 1-5 p.I. sind nicht beachtenswert verschieden vom Ausgangswert ( $p>$ $0,1)$; sie war am 1. Tag p.I. sogar vermindert (28\%). Das Kiemendeckelspreizen stieg wiederum an; am 2. Tag p.I. betrug die Zunahme $40 \%(\mathrm{p}<0,01)$.

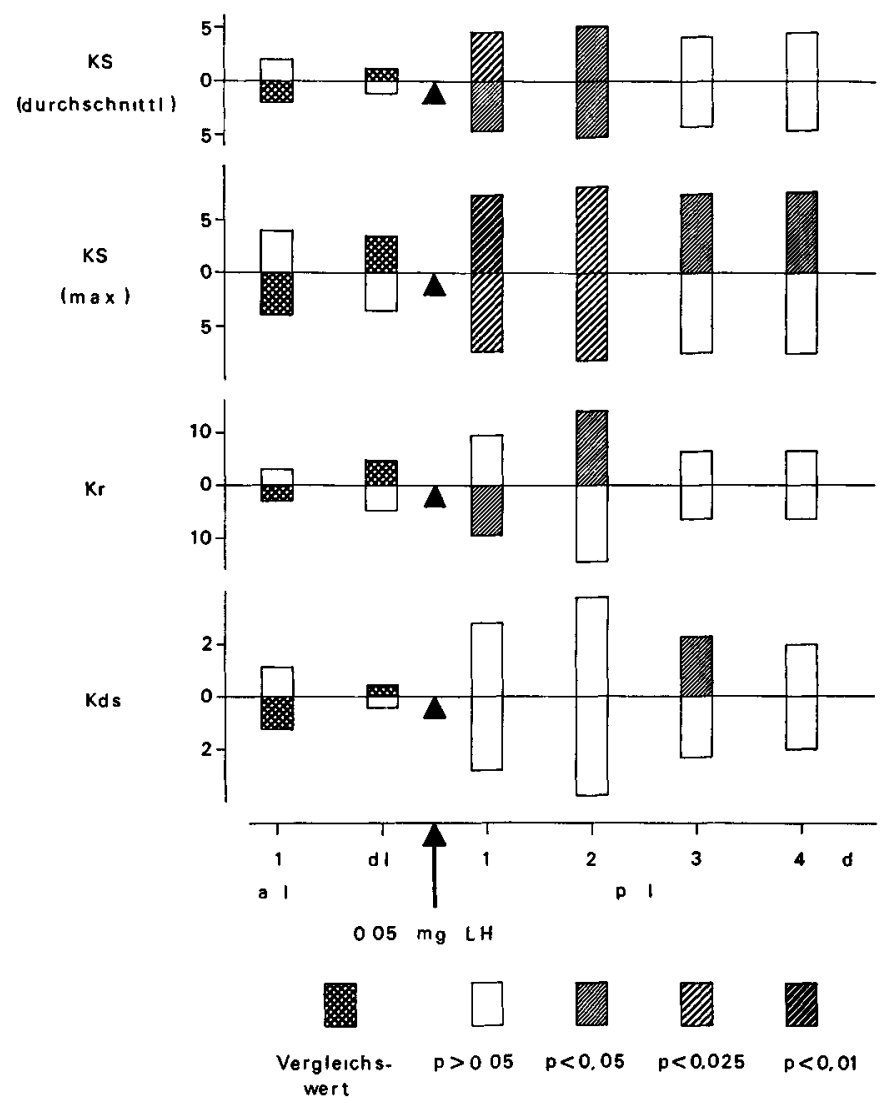

$A b b$. 4: Die Wirkung von injiziertem Luteinisierendem Hormon auf das Verhalten von Sonnenbarsch- $\hat{\partial} \hat{\partial}$, die mit Methallibur vorbehandelt worden waren $(n=4)$. Die Mengenangabe versteht sich pro Gramm Körpergewicht. $d I=$ Tag der Injektion. Sonst wie Abb.1

\section{Die Wirkung von exogenem Gonadotropin auf mit Methallibur gehemmte Tiere}

Im vorigen Abschnitt konnte ein Einfluß des Testosterons auf die aggressive Tendenz sowie auf das Kiemendeckelspreizen, das einen Konflikt zwischen aggressiver und Sexualtendenz anzeigt (KRAMER 1971), nicht aber auf das 
Sexualverhalten nachgewiesen werden. Daher spricht die hemmende Wirkung des Antigonadotropins Methallibur auf das Sexualverhalten (Abb. 1 und Tab. 1) für eine direkte Steuerung dieser Stimmung durch das gonadotrope Hormon der Hypophyse. Zur Klärung dieser Frage wurden $4 \hat{\partial} \hat{o}$ nach erfolgter Verhaltenshemmung durch Methallibur-Gaben ${ }^{4}$ ) mit Säugergonadotropin injiziert (siehe Abb. 4).

Am 2. Tag nach der LH-Injektion stieg die Sexualtendenz gegenüber dem Injektionstag im Mittel um $222 \%$ an $(p<0,05)$. Auch für das Kämpfen ergaben sich beachtenswerte Unterschiede: die durchschnittliche Kampfstärke stieg um $283 \%(p<0,025,1$. Tag p.I.) und $333 \%(p<0,05,2$. Tag p.I.), die maximale um $111 \%(\mathrm{p}<0,01,1$. Tag) und $134 \%(\mathrm{p}<0,025,2$. Tag) an. Das Kiemendeckelspreizen war am 3. Tag p.I. beachtenswert erhöht $(p<0,05)$ gegenüber dem Ausgangswert. Ein ähnliches Bild (bis auf Kiemendeckelspreizen) ergibt sich für den Vergleich dieser Werte mit denjenigen für den Vortag der LH-Injektion. Hierbei verschiebt sich bei Kreisen ein mit $p<0,05$ auffälliger Unterschied auf den 1. Tag p.I. (Dies hängt damit zusammen, daß ein Tier seinen Maximalwert schon 24 Std. nach der Injektion erreichte.)

\section{Die Wirkung von Reserpin}

In den vorausgehenden Abschnitten sind die humoralen Faktoren untersucht worden, die ein $\hat{\delta}$ in Bau-, Balz- und Laichstimmung versetzen. Durch das Zusammenwirken von Testosteron mit Gonadotropin wird das Ethogramm (siehe Kramer 1971, Abb. 11 a) auf wenige, spezifische Verhaltensweisen eingeengt, die der Nestbau-, Sexual- und aggressiven Tendenz unterstehen. Nichts ist bisher jedoch darüber ausgesagt worden, welcher Steuerungsmechanismus darüber entscheidet, welche der drei für die Fortpflanzungsphase charakteristischen und miteinander konkurrierenden Tendenzen sich im nächsten Augenblick in beobachtbarem Verhalten äußern wird. Die oben untersuchten Hormone können hierfür nicht in Betracht kommen, da sie einen Zeitbedarf von mindestens einem Tag haben, bevor sie fortpflanzungsspezifische Verhaltensweisen fördern. Oft löst bekanntlich ein neu auftretender Schlüsselreiz eine Verhaltensänderung aus (so wird z. B. ein einem isolierten, nestbauenden $\hat{o}$ hinzugesetztes $q$ sofort heftig angegriffen), in anderen Fällen aber muß die Verhaltensänderung auf innere Faktoren zurückgeführt werden (z. B. wenn aus dem zu vertreibenden und in die äußerste Beckenecke gejagten 9 ein Sexualobjekt wird). Ein weiteres Beispiel hierfür ist $\mathfrak{u}$. a. die immer wieder beim gemeinsamen Kreisen in der Nestgrube während des Ablaichens ungehemmt hervorbrechende Aggressivität des $\delta$ auch einem "willigen“ $q$ gegenüber. Es war daher nötig, nach einem zusätzlichen, kurzfristig wirkenden Steuerungssystem zu suchen. Als solches kommt nach neueren Untersuchungen der zentrale Catecholamin-Stoffwechsel in Betracht. Die Injektion des Psychopharmakons Reserpin, das die intraneuronalen Catecholamin-(Noradrenalin-, Dopamin- und Serotonin-)Speicher encleert, sollte diese Frage beantworten helfen.

4) Hemmung der Sexualtendenz gegenüber dem Ausgangswert: $95 \%(\mathrm{p}<0,001)$; des Kiemendeckelspreizens: $94 \%(\mathrm{p}<0,001)$; der durchschnittlichen Kampfstärke: $77 \%$ (p $<$ $0,001)$; der maximalen Kampfstärke: $53 \%$ ( $\mathrm{p}<0,05)$; Fegen und Jagen waren zu $100 \%$ gehemmt. 2 Tiere (Januar-März 1971) waren viermal mit 0,2 mg/g KG intramuskulär injiziert worden, die beiden anderen Tiere (Mai-Juli 1970) hatten Methallibur peroral erhalten (insgesamt $10,9 \mathrm{mg} / \mathrm{g} \mathrm{KG}$ ). Da bei der peroralen Substanzgabe der Depoteffekt entfällt, erhielten diese Tiere auch während des kritischen Versuchs täglich nach den Verhaltensmessungen Methallibur. 


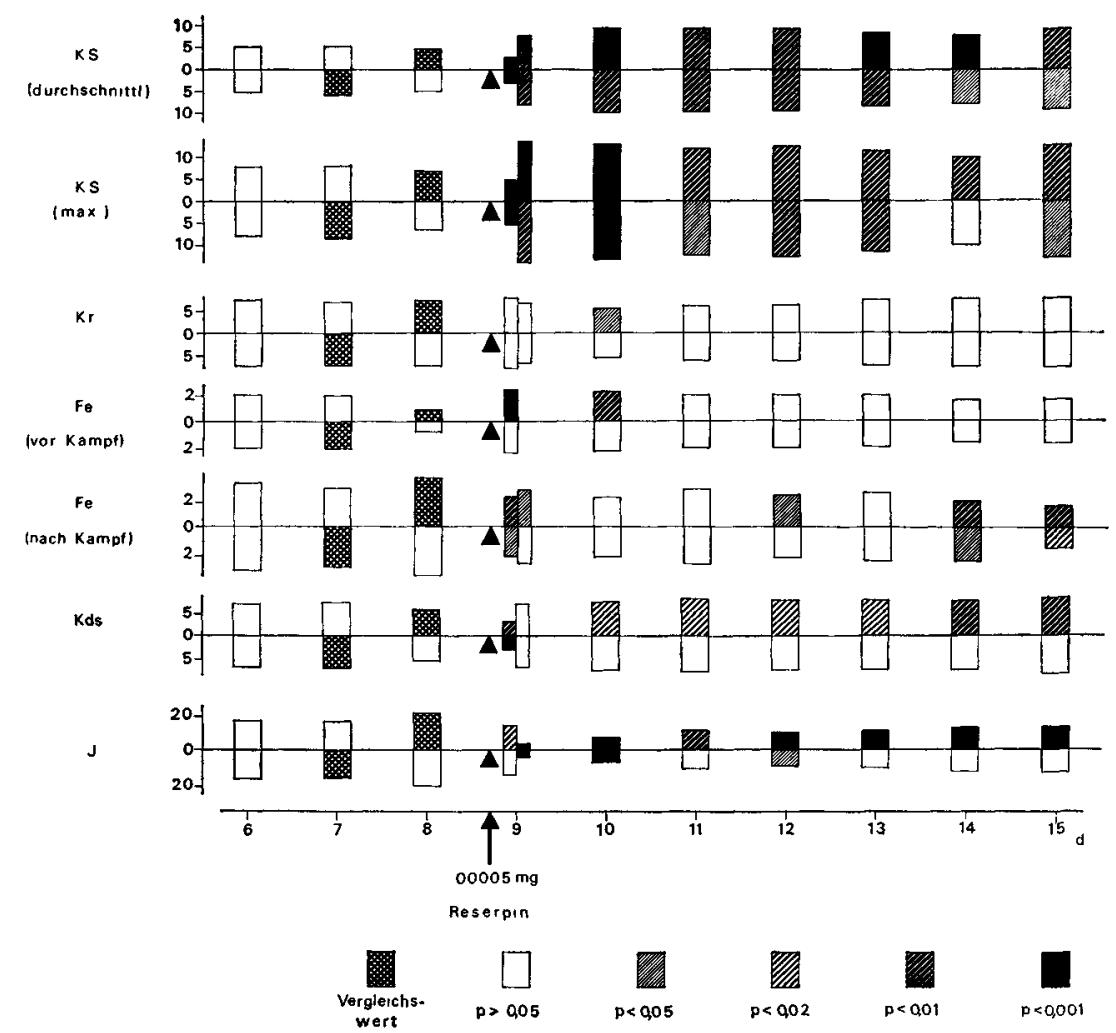

Abb.S: Die Wirkung von injiziertem Reserpin auf das Verhalten von Sonnenbarsch- $\hat{\phi} \hat{\sigma}$ $(\mathrm{n}=10$; September 1970). An Tag 9 sind Werte für 1 Std. und für 6 Std. p. I. angegeben. Sonst wie Abb. 1

Zehn balzende und nestbauende $\hat{\partial} \hat{t}$ (September 1970) erhielten $0,0005 \mathrm{mg}$ Reserpin/g KG intramuskulär injiziert (Abb. 5) ${ }^{5}$ ). Als signifikant sollen nur solche Differenzen angesehen werden, die von sowohl dem Tag wie dem Vortag der Injektion gesichert verschieden sind.

Besonders stark ist die Wirkung des Reserpins auf das Kampfverhalten; 1 Std. nach der Injektion sind die durchschnittliche und die maximale Kampfbereitschaft geringer $(p<0,001), 6$ Std. und später (Tage 10-15 mit Ausnahme des Tages 14 bei der maximalen Kampfstärke) gesichert größer. Für Sexualverhalten ergeben sich keine signifikanten Unterschiede, ebenso wenig für Fegen. Kiemendeckelspreizen ist 1 Std. p.I., Jagen 6 Std. sowie 1 Tag p.I. gesichert vermindert.

Im Tierversuch (Säuger und Vögel) ist bisher immer nur von einer sedativen Wirkung des Reserpins berichtet worden (v. SAINT PAUl 1965; Schildkraut und KetY 1967; BRUCKe, HoRNYKIEWICZ und SIGg 1969; Arzteprospekt ${ }_{n}$ Sedaraupin “ der Boehringer Mannheim GmbH.). Sie stieg mit wachsender Dosis (v. Saint Paul 1965). Cano Puerta (1959) fand einen sedativen Effekt bei Temperaturen, die für die Tiere (Betta splendens) wesentlich zu hoch $\left(30-38^{\circ} \mathrm{C}\right)$ oder wesentlich zu niedrig $\left(14-20^{\circ} \mathrm{C}\right)$ waren, nicht aber im Temperaturbereich von $24-25^{\circ} \mathrm{C}$. Auch MACHEMER (Diss. 1968) berichtet von einer sedativen Wirkung auf Macropodus opercularis- $\hat{\delta} \hat{\delta}$, die sich, ebenso wie die halbwüchsigen Segelflosser (Pterophyllum scalare) BLüMs (1968) für viele Tage schwärzlich verfärbten. Eine schwache Dunkelfärbung hielt bei Lepomis nur einige Stunden an, den sedativen Effekt konnte ich überhaupt nicht beobachten. Es war daher notwendig, auch höhere Dosen zu erproben.

5) Kontrollversuch: s. Abb. 2. Im Präparat „Sedaraupin“ ist Reserpin gelöst in einem Gemisch aus Wasser und 1,2-Propylenglykol (Auskunft der Fa. Boehringer). 
Drei der 10 Tiere erhielten 6 Tage p.I. die doppelte, 3 andere 7 Tage p.I. die vierfache und 2 weitere $\delta \hat{\delta} 13$ Tage p. I. die achtfache Dosis Reserpin. Auch nach diesen Injektionen hatte Reserpin keine sedative Wirkung; die Aggressivität stieg jedesmal schon 1 Std. p. I. an und blieb für 3-5 Tage erhöht (Einzelheiten siehe Kramer Diss. 1971).

\section{Die Wirkung von Chlorpromazin}

Chlorpromazin besitzt beim Menschen und bei Säugetieren eine sedative und tranquillierende Wirkung (BRücke, HoRNYKIEWICZ und Sigg 1969; Kuschinsky und LüllmanN 1970). Die gleiche Wirkung fand Cano Puerta (1959) bei Betta splendens, jedoch trat dieser Effekt, wie nach Reserpingaben (s. Kapitel Reserpin) nur bei zu hoher und zu niedriger, nicht aber bei normaler Temperatur auf und kann daher mit den hier geschilderten Ergebnissen nicht verglichen werden. Der Wirkungsmechanismus des Chlorpromazins ist noch nicht vollständig aufgeklärt, doch ist ein beschleunigter enzymatischer Abbau vor allem des Noradrenalin festgestellt worden (s. BRüCKE, HORNYKIEwICZ und SIGG 1969). Die durch Reserpin und ähnlich wirkende Pharmaka erhöhte Freisetzung von Catecholaminen in Säuger-Gehirnen wird durch Chlorpromazin verhindert. Dieser Reserpin-Chlorpromazin-Antagonismus sollte sich auch in entgegengesetzten Effekten auf das Verhalten äußern.

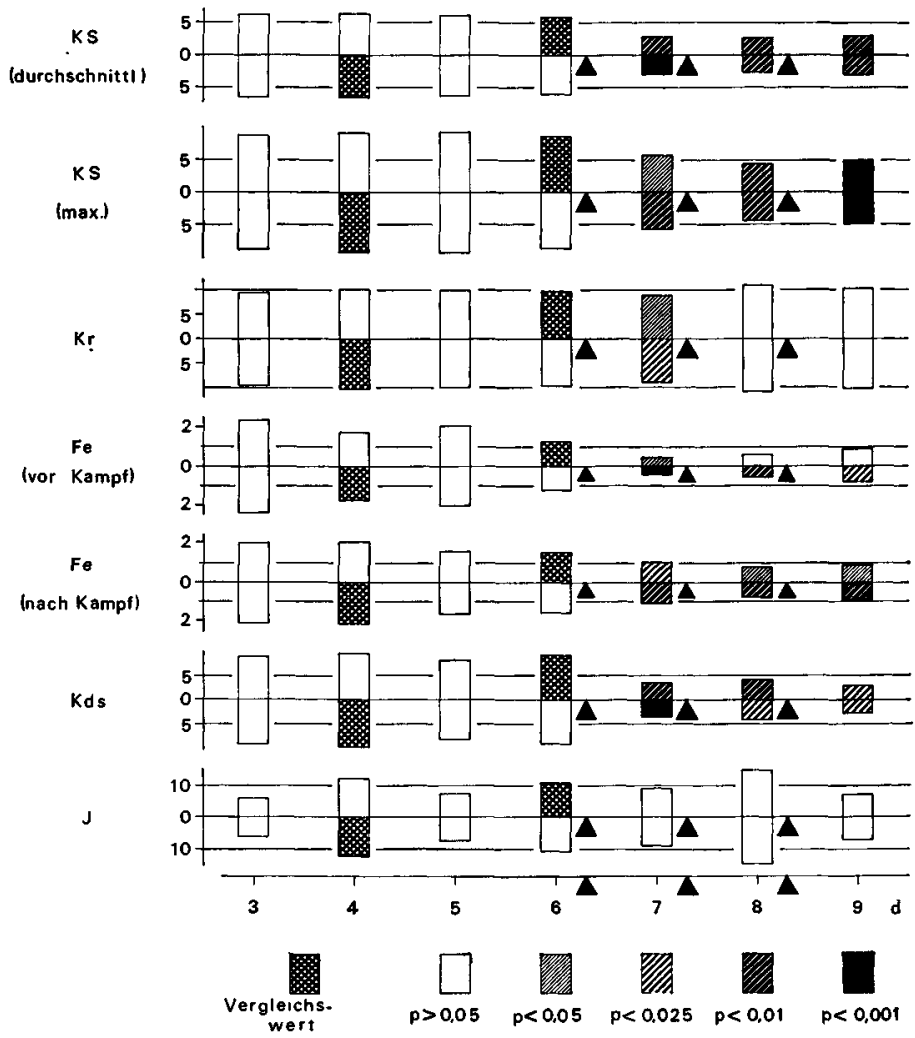

Abb.6: Die Wirkung von im Aquarienwasser gelöstem Chlorpromazin auf das Verhalten von Sonnenbarsch- $\hat{O} \delta \hat{~}(\mathrm{n}=5$; Juli 1970). Zugabe der vollen Substanzmenge $(0,125 \mathrm{mg} / \mathrm{l})$ an Tag 6, Erneuerung des Wassers und des Psychopharmakons je zur Hälfte an Tag 7 und 8. Sonst wie Abb. 1

Fünf balzenden und Nester bauenden $\hat{o} \hat{o}$ (Abb. 6) gab ich Chlorpromazin ins Aquarienwasser.

Gegenüber 2 Vergleichstagen vor der Behandlung waren die durchschnittliche und die maximale Aggressivität nach der Substanzzugabe signifikant ver- 
mindert. Für Kreisen ergaben sich keine gesicherten Unterschiede (jedoch ein geringfügiger, beachtenswerter Abfall am 1. Tag). Kiemendeckelspreizen war deutlich vermindert (Sicherung: $p<0,01$ am 1. Tag und $p<0,025$ an den beiden folgenden). Auch Fegen vor dem Kampf (am 1. Tag) und Fegen nach dem Kampf waren beachtenswert gehemmt. Für Jagen ergaben sich keine auffälligen Unterschiede.

\section{Histologische Untersuchung der Gonaden Methallibur-behandelter Fische}

$\mathrm{Da}$ unter natürlichen Lebensbedingungen Verhaltensweisen der Fortpflanzung synchronisiert mit dem Reifen der Gameten auftreten, ist zu erwarten, daß eine Hemmung dieser Verhaltensweisen mit regressiven Veränderungen an der Gonade einhergeht. PickFord und ATZ (1957), Dodd et al. (1960) sowie AHSAN (1966) haben die Wirkungen einer Hypophysektomie beschrieben; ein Gonadotropinhemmstoff müßte die gleichen histologischen Veränderungen hervorrufen. Bei Methallibur-behandelten Goldfischen (Carassius auratus), Stichlingen (Gaste-

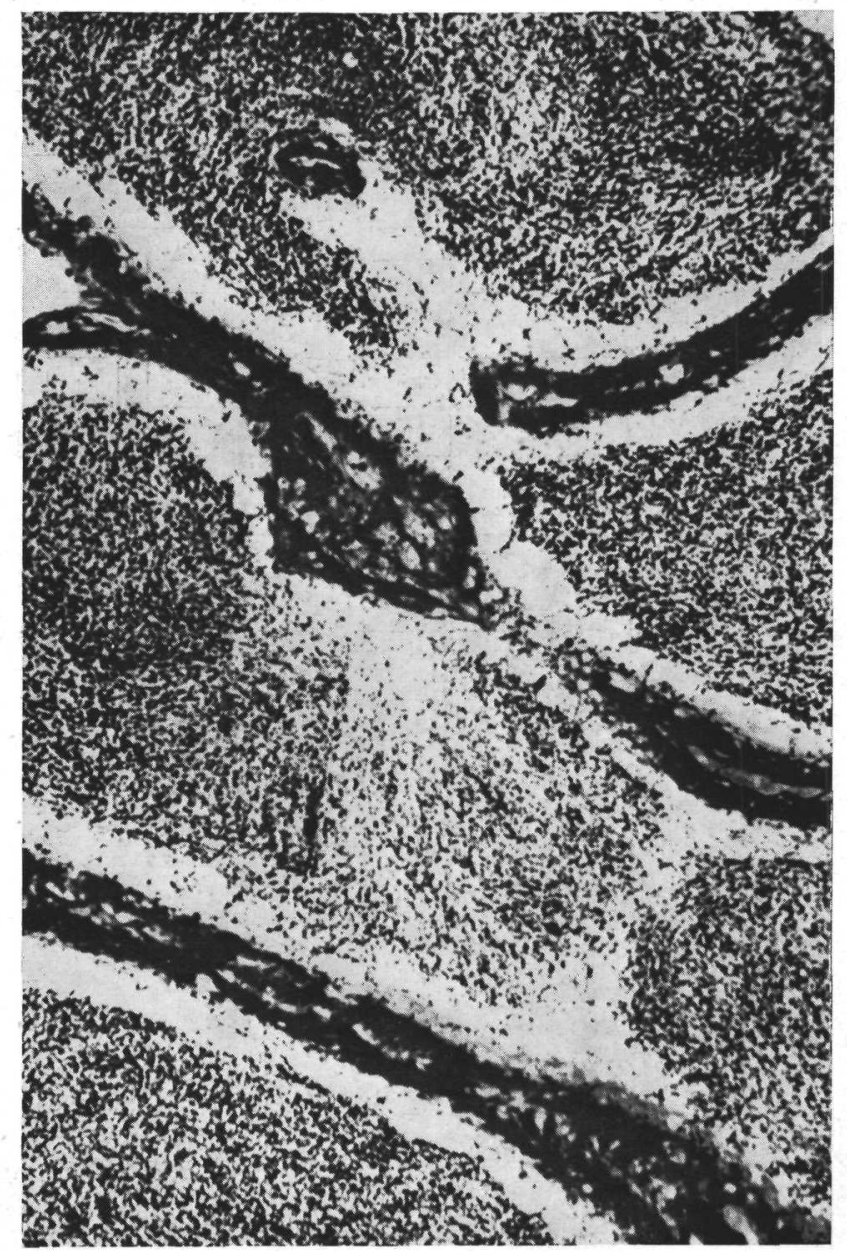

Abb.7: Prall mit Spermatozoen gefüllte Vasa efferentia des Hodens eines Kontrolltieres, das an 23 aufeinanderfolgenden Tagen (Juni 1970) peroral und anschließend $3 X$ in 2tägigem Abstand (beginnend an Tag 24) intramuskulär mit einer 10 Vol.\%igen wäßrigen PropylenglykolLösung behandelt worden war. Verabreichte Volumina/g KG gleichen den bei den Versuchstieren verwendeten. Original $\times 112,5$ 


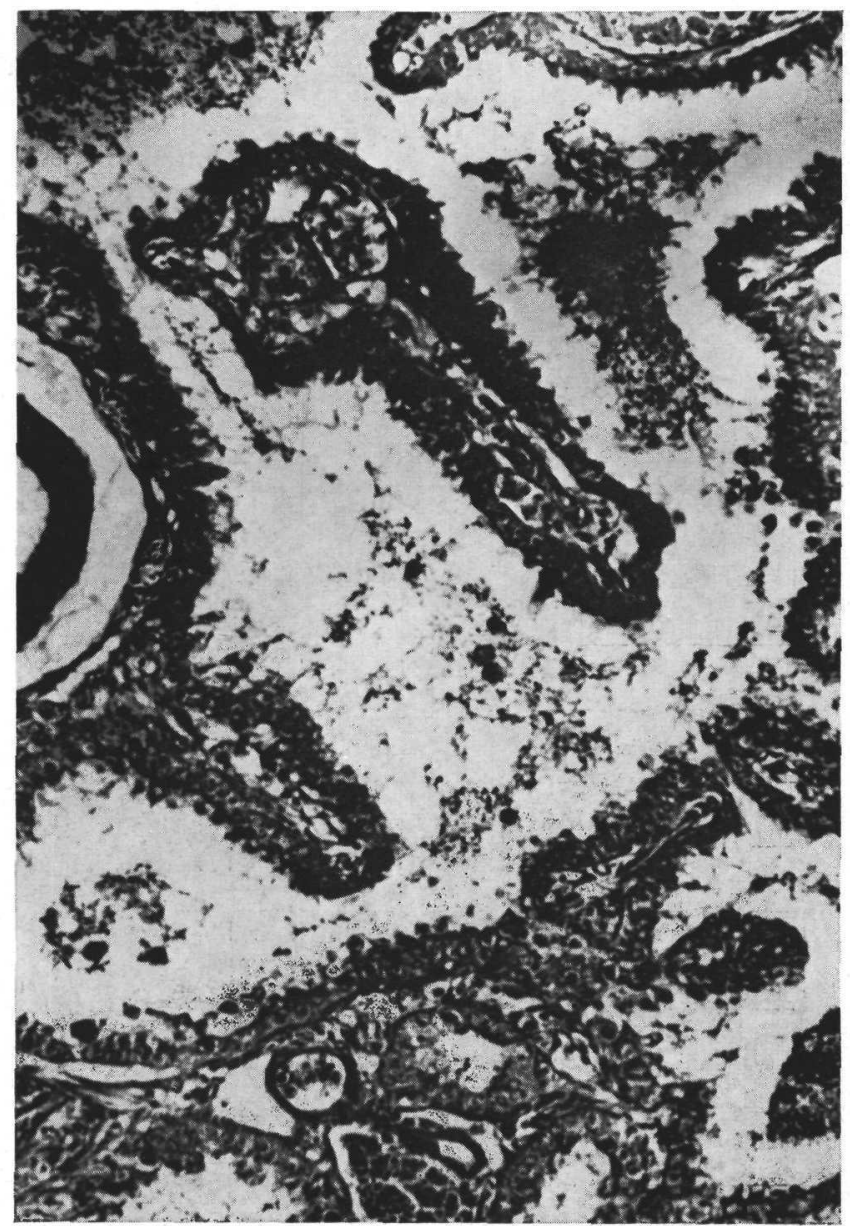

Abb.8: Vasa efferentia des Hodens eines Versuchstieres, das an 28 aufeinanderfolgenden Tagen peroral mit Methallibur (insgesamt 18,4 mg/g KG) behandelt worden war (Juni 1970). Original $\times 112,5$

rosteus aculeatus) und Cymatogaster aggregata ist dies nach HOAR, WIEBE und WAI (1967) und Wiebe $(1968,1969)$ der Fall.

Die weitlumigen Vasa efferentia der Hoden von Sonnenbarschen in Fortpflanzungsstimmung enthalten enorme Mengen von Spermatozoen (Abb. 7); in den Hodentubuli verläuft, wie man an der großen Zahl von Spermatocyten und Spermatiden erkennen kann (Abb. 9), lebhafte Spermiogenese. Spermatogonien sind selten.

Nach einer Methallibur-Behandlung (28 Tage) verringert sich die Weite der abführenden Kanäle, und die darin enthaltene Spermatozoenmenge ist erheblich vermindert (Abb. 8). Der Durchmesser der Hodentubuli, die nur noch wenige Spermatozoen enthalten, ist kleiner geworden; andere Teilungsstadien als Spermatogonien sind selten. Das $Z$ wischengewebe erscheint geschrumpft (Abb. 10).

Nach Pickford und ATz (1957), Dodd et al. (1960) und Ahsan (1966) sind die Reifeteilung und die sich anschließenden Zellteilungen gonadotropinabhängig; die sich mitotisch teilenden Spermatogonien werden von Gonadotro- 




Abb.9: Das gleiche Präparat wie in Abb. 7. Teilungsaktives spermatogenes Gewebe. Original $\times 450$

pinmangel nicht betroffen. Die hier dargestellten histologischen Veränderungen nach einer Methallibur-Behandlung entsprechen vollkommen denen, die nach Hypophysektomie erhalten worden sind; sie befinden sich auch in guter Übereinstimmung mit den Ergebnissen von HoAr et al. (1967) und WIEBE (1968, 1969).

\section{Diskussion}

Steroidhormone der Gonade und teilweise auch der Nebennierenrinde wirken über einen negativen Rückkopplungsmechanismus hemmend auf die Gonadotropin-Sekretion der Hypophyse ein (siehe NeumanN et al. 1967). Da ein Steroidhormon, Testosteron, in dieser Arbeit auf seine Verhaltenswirksamkeit hin überprüft werden sollte, war es notwendig, ein Nichtsteroid als Gonadotropinhemmstoff zu verwenden. Ein solcher ist Methallibur.

Die in der Literatur beschriebenen Wirkungen von Methallibur an Säugetieren und am Menschen wie Atrophie der Hoden und der akzessorischen Ge- 


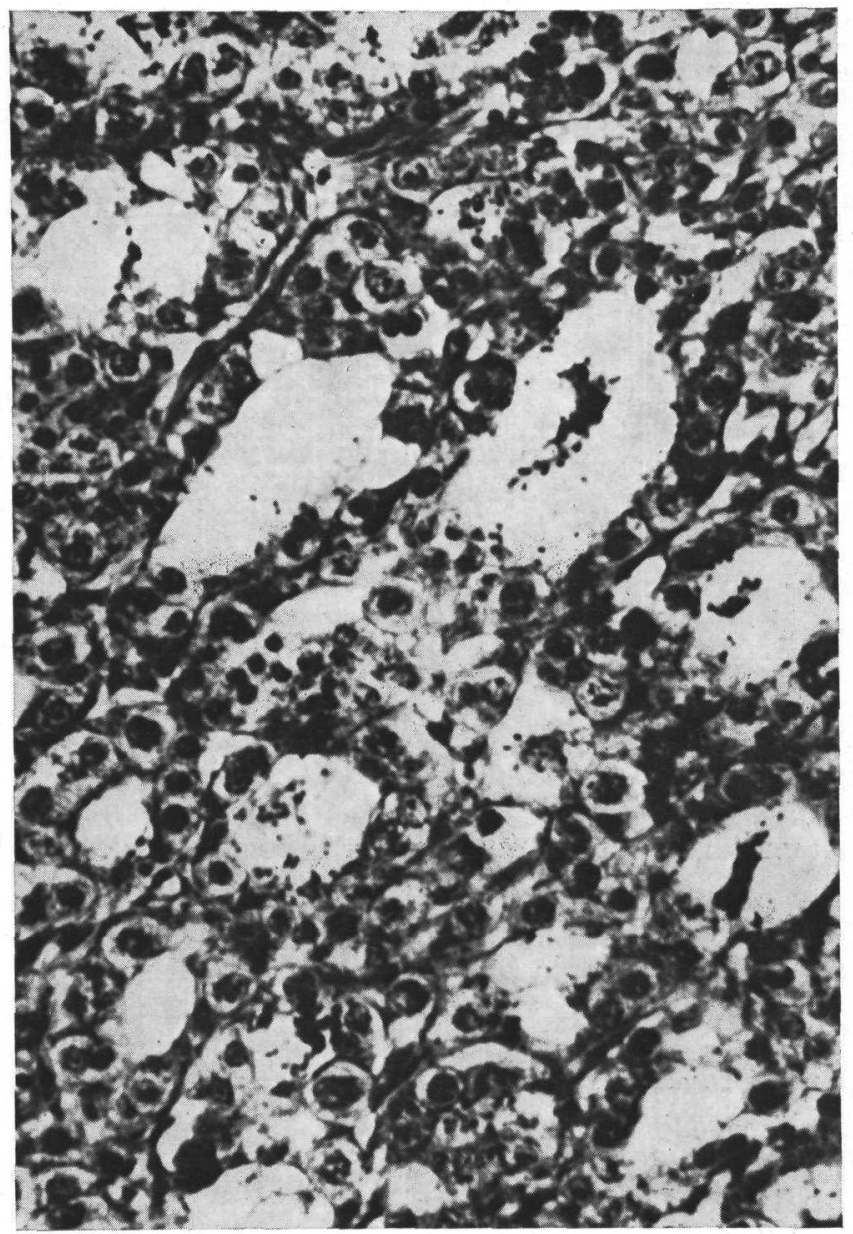

Abb. 10: Das gleiche Präparat wie in Abb. 8. Teilungsinaktives spermatogenes Gewebe. Original $\times 450$

schlechtsdrüsen und damit verbundene Sterilität, Hemmung des Östrus und der Follikelreifung in den Ovarien, sowie Abnahme des Gonadotropin-Gehaltes im Harn werden auf eine Hemmung der Gonadotropin-Synthese oder -Abgabe der Hypophyse zurückgeführt (PAGET et al. 1961; BELl et al. 1962; BRown 1963; Parkes 1963; Harper 1964; Walpole 1965; Polge 1965; Hemsworth et al. 1968; Skinner und Adams 1969; Skinner und Rowson 1969). Entsprechende Ergebnisse liegen für Hühner (Sykes 1964; El Jack und Sykes 1965; Mitchell 1967), eine Frosch-Art (KanAKaraJ und GANGADHARA 1967), sowie für 3 Knochenfischarten (Carassius auratus, Gasterosteus aculeatus und Cymatogaster aggregata) vor (HoAR et al. 1967; WIEBE 1968, 1969). GALOW (1969) konnte mit peroralen Methallibur-Gaben den Schaumnestbau des Kampffisches Betta splendens hemmen. Dieses Ergebnis führte Galow auf Androgen-Mangel zurück, der durch eine verminderte Gonadotropin-Ausschüttung zustande gekommen sein mußte.

Die in dieser Arbeit beschriebenen Wirkungen von Methallibur auf Sonnenbarsch- $\hat{o} \hat{o}$ sprechen ebenfalls für eine Hemmung der Gonadotropin-Ausschüttung der Hypophyse. Es zeigten sich regressive Veränderungen am histologischen 
Bild der Hoden (Abb. 8 und 10), die einer signifikanten Hemmung des Sexualverhaltens, Nestbauens, Kiemendeckelspreizens und Jagens (Abb. 1) sowie teilweise $^{6}$ ) auch der Kampfstärke (Tab. 1) entsprachen. Bei den Kontrolltieren (Abb. 2) wurden keine signifikanten Unterschiede registriert, auch das histologische Bild der Gonaden erscheint unverändert (Abb. 7 und 9). Im Einklang hiermit steht die Beobachtung, daß mit Methallibur behandelte $\hat{\delta} \hat{\delta}$ fächelten (S. 358). Diese Brutpflegeverhaltensweise kann bei Sonnenbarschen durch kombinierte Säugerprolactin-Testosteron-Gaben ausgelöst werden (KRAMER 1969, 1971; hierzu siehe auch: FIEDLER 1962; BlüM und FIEDLER 1964 und 1965; BlüM 1966; Bartmann Diss. 1968). Zwischen Gonadotropin und Prolactin besteht nach Riddle et al. (1933) sowie BAtes et al. (1935) und Blüm (1966) ein Antagonismus; eine Hemmung der Gonadotropin-Ausschüttung mußte eine vermehrte Prolactin-Sekretion und damit Fächeln zur Folge haben. Die Tiere stellten diese Verhaltensweise nach wenigen Tagen wieder ein, offenbar weil der Gonadotropin-Mangel mit einer gewissen Verzögerung auch den AndrogenSpiegel senkte ${ }^{7}$.

Die geschilderten Ergebnisse zeigen die (schon bekannte) allgemeine $\mathrm{Ab}$ hängigkeit des Fortpflanzungsverhaltens (der Bau-Balz-Laichphase) von Gonadotropin und die Möglichkeit, dieses Verhalten durch ein Antigonadotropin zu hemmen; es läßt sich hieraus noch nicht entnehmen, ob Gonadotropin eine direkte Verhaltenswirksamkeit besitzt, oder ob eine Sekundärwirkung über die Gonade vorliegt. Anschließende Versuche an Tieren, die mit Methallibur vorbehandelt worden waren und dann mit Testosteron oder LH injiziert wurden, sollten diese Frage klären.

Testosteron steigerte signifikant die Kampfstärke und das Kiemendeckelspreizen (Abb. 3); Gonadotropin förderte vor allem Sexualverhalten, aber auch die Kampfstärke und das Kiemendeckelspreizen sind auffällig erhöht (Abb. 4).

Jagen ließ sich, nachdem es immer sehr schnell bei allen Versuchstieren durch Methallibur gehemmt worden war, nicht wieder mit den verwendeten Hormonen auslösen. Diese Handlung ist wie Kiemendeckelspreizen eine Verhaltensweise, die einen Konflikt zwischen aggressiver und Sexualtendenz anzeigt (KRAMER 1971). Damit Jagen gezeigt wird, muß der Konflikt zwischen aggressiver und Sexualtendenz sehr intensiv sein. Dies kann man u. a. daran erkennen, daß es in der Regel nur in der 1. Minute des fünfminüitigen BalzTests, in der sich das Schwergewicht von der aggressiven auf die Sexualtendenz verlagert, ausgeführt wird. Obwohl Gonadotropin die aggressive und die Sexualtendenz gleichzeitig steigerte, reichte die Intensität des Konfliktes zwischen beiden Stimmungen aber offenbar nicht aus, um Jagen auszulösen.

Nestbauverhalten konnte weder durch Testosteron- noch durch Gonadotropin-Injektionen gefördert werden, nachdem es durch das Antigonadotropin vollständig gehemmt worden war; möglicherweise steht dies im Zusammenhang mit Degenerationen im Gehirn, die Methallibur schon 6 Tage nach einer einmaligen Injektion bewirkt (FIEDLER, persönl. Mitteilung). Die in der Einleitung dargestellten Befunde (SMITH, 1969; KRAMER 1969, 1971) lassen jedoch keinen Zweifel daran, daß Nestbauverhalten wenigstens überwiegend durch Androgen gesteuert wird.

6) Hinweis auf jahreszeitliche Abhängigkeit s. S. 358.

7) Auf eine durch Methallibur bedingte vermehrte endogene Prolactin-Sekretion weist auch der Befund Galows (1969) hin, nach dem die Schleimzellenzahl in der Schuppenepidermis von Betta splendens nach einer Methallibur-Behandlung deutlich erhöht war. Das gleiche Ergebnis erhielt man nach intramuskulären Injektionen von Säuger-Prolactin (BLÜM und FIEDLER 1964, 1965; MACHEMER und Fiedler 1965; BlüM 1966; MACHemer 1971). 
SMITH (1970) fand weder einen Einfluß von Androgen noch von (menschlichem Chorion-)Gonadotropin auf die Aggressivität von Sonnenbarschen, nach ihm ist nur die Wassertemperatur von Bedeutung. Lediglich die letzte Aussage ist sicher richtig, gegen die beiden ersteren sprechen die oben dargestellten Befunde. Die Erklärung für diese Diskrepanz ist hauptsächlich in der unterschiedlichen ethologischen Methodik begründet (siehe auch KRAMER 1971). SMITH verwendete Kiemendeckelspreizen, Schwanzschlagen und „fluttering“ (Stoßen nach einem Beckennachbarn hinter einer transparenten Kunststofftrennscheibe) als Meßgrößen für aggressives Verhalten. Die ersten beiden Verhaltensweisen sind nur zum Teil aggressiv motiviert, sie zeigen einen Konflikt an (KRAMER 1971). Aber auch „fluttering“ ist als Meßgröße für aggressives Verhalten solange nicht geeignet, bis gezeigt wurde, daß die mit der Trennscheibe bewirkte Störung des gesamten Verhaltensablaufs die Meßwerte nicht ändert (in eigenen Beobachtungen war dies jedoch der Fall). Im Falle des Gonadotropin-Versuchs kommt noch ein weiterer Unterschied hinzu: Choriongonadotropin ist dem Sonnenbarsch-Gonadotropin chemisch sicher unähnlicher als das in dieser Arbeit verwendete Luteinisierende Hormon aus Hypophysen.

Ein weiteres Steuerungssystem, das Fortpflanzungsverhalten beeinflußt, stellt wahrscheinlich der zentrale Catecholaminstoffwechsel dar; dem Noradrenalin kommt offenbar für aggressives Verhalten eine besondere Bedeutung zu (Schildkraut und KeTY 1967). Dies wird durch die Ergebnisse dieser Arbeit bestätigt, in der injiziertes Reserpin die Kampfstärke der Sonnenbarsche über eine Reihe von Tagen signifikant erhöhte (Abb. 5); ein sedativer Effekt trat auch nach Dosissteigerung nicht ein. Diese Beobachtung erscheint unvereinbar mit vielen Mitteilungen in der Literatur (siehe S. 362), nach denen Reserpin eine sedative Wirkung besitzt, die (z.B. v. SaInt-Paul 1965) mit der Dosis steigt. In diesem Zusammenhang sind die Ausführungen von KUSCHINSKY und LüLLMANN (1970) von Bedeutung: nach hohen Dosen Reserpin tritt zunächst eine sympathomimetische Reaktion auf, die einer intravenösen Infusion von Noradrenalin gleicht, und die durch die plötzliche Freisetzung dieses Amins aus den Speichergranula der Nervenendigungen ausgelöst wird. Einen sedativen Effekt erhält man nur, wenn die Behandlung "einschleichend" begonnen wird, so daß pro Zeiteinheit nur unterschwellige Mengen Noradrenalin frei werden. Es ist daher anzunehmen, daß die in dieser Arbeit verwendeten Dosen (die denen der zitierten Arbeiten entsprechen) beim Sonnenbarsch überschwellig sind.

Die Erklärung dafür, daß die Wirkung einer Reserpin-Injektion mehrere Tage anhält, liegt in einer Schädigung der Noradrenalin-speichernden Granula, die erst wieder in Kernnähe aus endoplasmatischem Retikulum gebildet werden und den ganzen Neuriten zur Synapse hinunterwandern müssen (KuschINSKY und LüLLMANN 1970).

Chlorpromazin, das die Wirkung ausgeschütteten Noradrenalins verhindert, hatte auf die Kampfstärke der Sonnenbarsche den entgegengesetzten Effekt: es wurden signifikant niedrigere Werte gemessen (Abb.6). Sexualverhalten erscheint unvermindert, Nestbauen und Kiemendeckelspreizen sind beachtenswert gehemmt. Die kurzfristige Steuerung der aggressiven und der Nestbaustimmung ist demnach noradrenerg. MARRONE et al. (1966) teilen mit, daß im Wasser gelöstes Noradrenalinbitartrat bei Betta splendens einen signifikanten Anstieg des Flossen- und Kiemendeckelspreizens sogar in vacuo ausgelöst habe; dieser Befund wird allerdings nicht von BAENNINGER (1968) bestätigt, der eine signifikante Erniedrigung fand. Noradrenalin kann die Blut-Hirn-Schranke nicht passieren (SCHILDKRAUT und KETY 1967).

Die geschilderten Ergebnisse lassen vermuten, daß die Sexualhormone (mit den oben dargestellten unterschiedlichen Schwergewichten) lediglich die Wahr- 
scheinlichkeit für das Auftreten von Verhaltensweisen der Fortpflanzung erhöhen, während Catecholamine bei der aktuellen Ausführung steuernd eingreifen.

\section{Zusammenfassung}

1. Die intramuskuläre Injektion des Antigonadotropins Methallibur (I.C.I. $33828 ; 3 \times 0,2 \mathrm{mg} / \mathrm{g}$ Körpergewicht in 2tägigem Abstand) hemmte das Sexualverhalten, das Nestbauen und zwei Verhaltensweisen des Konfliktes zwischen aggressiver und Sexualtendenz, das Kiemendeckelspreizen und das Jagen signifikant (d.h. $\mathrm{p}<0,01$ ) gegenüber dem Ausgangswert (Abb. 1). Keine solchen Unterschiede ergaben sich bei den Kontrolltieren (Abb. 2).

2. Nur die mit Methallibur injizierten Versuchstiere fächelten $a b$ dem 4. bis 5. Tag p. I. für mehrere Tage (Brutpflegeverhalten).

3. Die Hoden von Tieren, die über 4 Wochen mit Methallibur behandelt worden waren, weisen starke regressive Veränderungen auf (Abb. 7-10). Sie gleichen denen, die bei hypophysektomierten Tieren beschrieben worden sind.

4. Intramuskulär injiziertes synthetisches Testosteron $(0,06 \mathrm{mg} / \mathrm{g} \mathrm{KG})$ steigerte innerhalb von $2-3$ Tagen signifikant die Kampfstärke und das Kiemendeckelspreizen von Tieren, die mit Methallibur vorbehandelt worden waren (Abb. 3).

5. Intramuskulär injiziertes Säuger-LH (NIH; $0,05 \mathrm{mg} / \mathrm{g} \mathrm{KG)} \mathrm{förderte}$ innerhalb von $1-3$ Tagen beachtlich (d.h. $p<0,05)$ das Sexualverhalten, die Kampfstärke und das Kiemendeckelspreizen von Tieren, die mit Methallibur vorbehandelt worden waren (Abb. 4).

6. Intramuskulär injiziertes Reserpin $(0,0005 \mathrm{mg} / \mathrm{g} \mathrm{KG})$, das die in den intraneuronalen Speichergranula enthaltenen Catecholamine Noradrenalin, Dopamin und Serotonin ausschüttet, steigert $6 \mathrm{Std}$. p. I. über eine Reihe von Tagen hinweg signifikant die Aggressivität. Auch nach der doppelten, der vierfachen und der achtfachen Dosis trat kein sedativer Effekt auf (Abb. 5).

7. Im Aquarienwasser gelöstes Chlorpromazin $(0,125 \mathrm{mg} / \mathrm{l})$, das die Wirkung ausgeschütteten Noradrenalins verhindert, verminderte die Kampfstärke signifikant; auch Nestbauen und Kiemendeckelspreizen waren beachtenswert erniedrigt. Ungehemmt war die Sexualtendenz (Abb. 6).

8. Die Ergebnisse unter 6. und 7. zeigen, daß die aktuelle Steuerung der aggressiven und der Nestbau-Stimmung noradrenerg ist. Sie weisen auf den zentralen Catecholaminstoff wechsel als ein kurzfristig wirkendes Steuerungssystem für Fortpflanzungsverhalten hin.

\section{Summary}

Chemical Compounds in the Nest-Building, Sexual and Aggressive Behaviour in the Pumpkin-Seed Sunfish Lepomis gibbosus (L.) (Centrarchidae, Teleostei)

1. Intramuscular injection $(3 \times 0.2 \mathrm{mg} / \mathrm{g}$ body weight in 2-day intervals) of the antigonadotropin Methallibure (I.C.I. 33 828) decreased the sexual and suppressed the nest-building tendencies significantly (i. e. p $<0.01$ ). Two motor patterns indicating a conflict between the aggressive and the sexual tendencies, viz. opercular spreads and "leading" (Jagen), diminished significantly (Fig. 1). No such effects were recorded in the control animals (Fig. 2). 
2. Fanning (parental behaviour) was performed by the animals treated with Methallibure $4-5$ days p.i. for several days. This was not observed in the control animals.

3. The process of gametogenesis in the testes of animals pretreated for 4 weeks with Methallibure was suspended, an effect well known in hypophysectomized animals (Fig. 7-10).

4. In animals pretreated with Methallibure, intramuscular injections of synthetic testosterone $(0.06 \mathrm{mg} / \mathrm{g} \mathrm{b}$.w.) stimulated the intensity of aggressive behaviour and the frequency of opercular spreads significantly within $2-3$ days p.i. (Fig. 3).

5. In animals pretreated with Methallibure, intramuscular injections of mammalian LH (NIH, $0.05 \mathrm{mg} / \mathrm{g} \mathrm{b.w.)} \mathrm{increased} \mathrm{the} \mathrm{frequency} \mathrm{of} \mathrm{sexual} \mathrm{and}$ aggressive motor patterns as well as the frequency of opercular spreads within $1-3$ days p.i. ( $\mathrm{p}<0.05$, Fig. 4 ).

6. Intramuscular injections of Reserpine $(0.0005 \mathrm{mg} / \mathrm{g} \mathrm{b.w.),} \mathrm{which} \mathrm{depletes}$ the intraneuronal storage granules of catecholamines, stimulated the aggressivity of the animals significantly within $6 \mathrm{hrs}$. p.i. for a period of several days (Fig. 5). Even a two-, a four-, and an eightfold dose of Reserpine did not produce sedation.

7. Chlorpromazine $(0.125 \mathrm{mg} / \mathrm{l}$ dissolved in the aquarium water), which inhibits the action of released norepinephrine, depressed the aggressivity significantly, as well as nest-building behaviour and opercular spreads ( $\mathrm{p}<0.05$ ). The sexual tendency remained unaffected (Fig. 6).

8. The last-mentioned observations (6. and 7.) demonstrate that the actual control of the aggressive and the nest-building tendencies is mediated by norepinephrine.

\section{Literaturverzeichnis}

AHSAN, S. N. (1966): Effects of gonadotropic hormones on male hypophysectomized lake chub, Couesius plumbens. Can. J. Zool. 44, 707-717 - Baenninger, R. (1968): Catechol amines and social relations in Siamese fighting fish. Anim. Behav. 16, 442-447 - BartmanN, W. D. (1968): Die Maulbrutpflege bei Buntbarsch-Weibchen (Cichlidae, Teleostei) und ihre endokrinen Grundlagen. Diss. nat. Fak. d. J. W. Goethe-Universität, Frankfurt/M. - BATES, R. W., E. L. LAHR und O. RIDDLE (1935): The cross-action of prolactin and follicle stimulating hormone on the mature ovary and sex accessories of fowl. Amer. J. Physiol. 3, 361-368 Bell, E. T., J. B. Brown, K. Fotherby, J. A. Loraine und J. S. Robson (1962): The effect of derivatives of dithiocarbamoylhydrazine on hormone excretion in postmenopausal women. J. Endocrinol. 25, 221-231 - BLÜM, V. (1966): Zur hormonalen Steuerung der Brutpflege einiger Cichliden. Zool. Jb. Physiol. 72, 264-290 - Bü̈M, V. (1968): Die Auslösung des Laichreflexes durch Reserpin bei dem südamerikanischen Buntbarsd Pterophyllum scalare. Z. vergl. Physiol. 60, 79-81 - BlüM, V., und K. FIEdLer (1964): Der Einfluß von Prolaktin auf das Brutpflegeverhalten von Symphysodon aequifasciata axelrodi L. P. Schultz (Cichlidae, Teleostei). Naturwiss. 51, 149-150 - BLüM, V., und K. FiedLer (1965): Hormonal control of reproductive behaviour in some Cidhlid fish. Gen. Comp. Endocrinol. 5, 186-196 - Brown, P.S. (1963): Observations on a dithiocarbamoylhydrazine as an inhibitor of pituitary gonadotrophic activity. J. Endocrinol. 26, 425-436 - BR ÜCKE, F. Th. v., O. HoRNYKIE WICZ und E. B. SIGG (1969): The pharmacology of psychotherapeutic drugs. Heidelberg Science Library, Springer Verlag - CANo Puerta, G. (1959): Die Wirkung von Tranquilizern auf tropische Fische. Arch. int. Pharmacodyn. 121, 404-414 . Cavalti-Sforza, L. (1969): Biometrie. Grundzüge biologisch-medizinischer Statistik. G. Fischer-Verlag Stuttgart - DonD, J. M., P. J. EvenNeTT und C. K. GODDARD (1960): Reproductive endocrinology in cyclostomes and elasmobranchs. Symp. Zool. Soc. London 1, 77-103 - EIBL-EIBESFELDT, I. (1967): Grundriß der vergleichenden Verhaltensforschung. R. Piper \& Co. Verlag München - EL JACK, M. H., und A. H. SyKes (1965): Effect of a dithiobiurea derivative on semen characteristics and fertility in the fowl. J. Reprod. Fert. 10, 79 - Fiedrer, K. (1962): Die Wirkung von Prolactin auf das Verhalten des Lippfisches Crenilabrus ocellatus (Forskål). Zool. Jb. Physiol. 69, 609-620 - FiEdLER, K. 
(1964): Verhaltensstudien an Lippfischen der Gattung Crenilabrus (Labridae, Perciformes). Z. Tierpsychol. 21, 521-591 - FIEDLER, K. (1967): Ethologische und neuroanatomische Auswirkungen von Vorderhirnexstirpationen bei Meerbrassen (Diplodus) und Lippfischen (Crenilabrus, Perciformes, Teleostei). J. Hirnforschung 9, 481-563 • Galow, J. (1969): Der Nestbau des Kampffisches Betta splendens R. (Anabantidae) und seine hormonale Beeinflussung. Staatsexamensarbeit in Biologie, nat. Fak. d. J. W. Goethe-Univ., Frankfurt/M. - Harper, M. J. K. (1964): Effects of 1- $\alpha$-methylallylthiocarbamoyl-2-methylthiocarbamoyl-hydrazine (I.C.I. 33, 828 ) on early pregnancy in the rat. J. Reproduction Fertility 7, 211-220 - HeILIGeNBerg, W. (1963): Ursachen für das Auftreten von Instinktbewegungen bei einem Fische (Pelmatochromis subocellatus kribensis Boul., Cichlidae). Z. vergl. Physiol. 47, 339-380 - HemsworTH, B. N., H. JACKSON und A. L. WALPOLE (1968): Effect of the pituitary inhibitor I.C.I. 33, 823 (Methallibure) on the reproductive system of the male rat. J. Endocrinol. 40, 275-284 - HOAR, W. S., J. WIEBE und E. H. WAI (1967): Inhibition of the pituitary gonadotropic activity of fishes by a dithiocarbamoylhydrazine derivative (I.C.I. 33,828). Gen. Comp. Endocrinol. 8, 101-109 - KanakaraJ, S. R., und N. S. Gangadhara (1967): Effect of a dithiocarbamoylhydrazine derivative (ICI 33,828) on spawning in the Skipper Frog, Rana cyanopblyctis Schneider. Gen. Comp. Endocrinol. 8, 72-74 • KRAMER, B. (1969): Zur hormonalen Steuerung von Verhaltensweisen der Fortpflanzung beim Sonnenbarsch Lepomis gibbosus (L.) (Centrarchidae, Teleostei). Diplomarbeit Naturwiss. Fak. d. Univ. Frankfurt/M. - KRAMER, B. (1971a): Zur hormonalen Steuerung von Verhaltensweisen der Fortpflanzung beim Sonnenbarsch Lepomis gibbosus (L.) (Centrarchidae, Teleostei). Z. Tierpsychol. 28, 351-386 - Kramer, B. (1971b): Faktoren, welche lang- und kurzfristig Nestbau-, Sexual- und Kampfverhalten beim Sonnenbarsch Lepomis gibbosus (L.) (Centrarchidae, Teleostei) bestimmen. Diss. nat. Fak. d. J. W. Goethe-Univ. Frankfurt/M. - KrAmer, B. (1972): Behavioural Effects of an Antigonadotropin, of Sexual Hormones, and of Psychopharmaka in the Pumkinseed Sunfish, Lepomis gibbosus (Centrarchidae). Experientia 28, 1195 • Kramer, B., W. Molenda und K. Fiedler (1969): Behavioural Effect of the Antiandrogen cyproterone acetate (Schering) in Trlapia mossambica and Lepomis gibbosus. Gen. Comp. Endocrinol. 13, 515 - Kuschinsky, G., und H. Lüllmann (1970) Kurzes Lehrbuch der Pharmakologie. Georg Thieme Verlag, Stuttgart - Machemer, L. (1968): Der Schaumnestbau beim Paradiesfisch, Macropodus opercularis L. (Anabantidae, Teleostei), und seine hormonale Kontrolle. Diss. Naturwiss. Fak. Universität Frankfurt/M. - MAchemer, L. (1971): Synergistische Wirkung von Säuger-Prolaktin und Androgen beim Schaumnestbau des Paradiesfisches, Macropodus opercularis L. (Anabantidae). Z. Tierpsychol. 28, 33-53 - Machemer, L., und K. FiedLer (1965): Zur hormonalen Steuerung des Schaumnestbaues beim Paradiesfisch, Macropodus opercularis L. (Anabantidae, Teleostei). Naturwiss. 52, 648-649 - Marrone, R.L., S. L. PraY und C. C. Bridges (1966): Norepinephrine elicitation of aggressive display responses in Betta splendens. Psychol. Sci. 5, 207-208 - Mrtchell, M. E. (1967): The effect of avian gonadotrophin precipitate on pituitary-deficient hens. J. Reprod. Fert. 14, 257-263 - NEUMANN, F., W. ELGER und R. v. BERSWORDT-W ALLRABE (1967): Intersexualität männlicher Feten und Hemmung androgenabhängiger Funktionen bei erwachsenen Tieren durch Testosteronblocker. Disch. Med. Wschr. 8, 360-366 - Paget, G. E., A. L. Walpole und D. N. RichardsoN (1961): Non-steroidal inhibitors of pituitary gonadotrophic function. Nature (London) 192, 1191 - PARKES, A. S. (1963): Review of work with ICI compound 33,828: a nonsteroidal pituitary inhibitor. J. Reprod. Fertil. 5, 459-460 - Pickford, G. E., und J. W. Atz (1957): The physiology of the pituitary gland of fishes. New York Zoological Society, New York POLGE, C. (1965): Effective synchronization of oestrus in pigs after treatment with ICI 33828. Vet. Rec. 77, 232 - RENSCH, B., und G. DúcKER (1966): Verzögerung des Vergessens erlernter visueller Aufgaben bei Tieren durch Chlorpromazin. Pflügers Archiv 289, 200-214 - RIDDLe, O., R. W. BATES und S. W. DYKshorN (1933): The preparation, identification and assay of prolactin - a hormone of the anterior pituitary. Amer. J. Physiol. 105, 191 - RomeIs, B. (1968): Mikroskopische Technik, 16. Aufl. R. Oldenbourg Verlag München-Wien - SAINT PAUL, U. v. (1965): Einfluß von Pharmaka auf die Auslösbarkeit von Verhaltensweisen durch elektrische Reizung. Z. vergl. Physiol. 50, 415-446 - Schild kraut, J. J., und S. S. KetY (1967): Biogenic amines and emotion. Science 156, 21-30 - Skinner, J. D., und C. E. AdAMs (1969): Some effects of a single injection of chlormadinone acetate or methallibure on sexual function in the pubescent male animal. II. The rabbit. J. Reproduct. Fertil. 20, 105-110 • SkINNER, J. D., und L. E. Rowson (1969): Some effects of a single injection of dhlormadinone acetate or methallibure on sexual function in the pubescent male animal. I. The ram. J. Reproduct. Fertil. 20, 95-104 - SMITH, R. J. F. (1969): Control of prespawning behaviour of sunfish (Lepomis gibbosus and L.megalotis). I. Gonadal Androgen. Anim. Behav. 17, 279-285 - Smith, R. J. F. (1970): Control of prespawning behaviour of sunfish (Lepomis gibbosus and L.megalotis). II. Environmental Factors. Anim. Behav. 18, 575-587 - SyKes, A. H. (1964): Some actions of an anti-fertility compound in the fowl. Vet. Rec. 76, 393-394. Tulloch, M. I., J. CRooks und P. S. BROWN (1963): Inhibition of thyroid function by a dithiocarbamoylhydrazine. Nature 199, 288-289 - Walpole, A. L. (1965): Non-steroidal agents inhibiting 
pituitary gonadotrophic function. In: "A symposium on agents affecting fertility" (C. R. Austin and J. S. Perry eds.), Churchill, London - WiebE, J. P. (1968): Inhibition of pituitary gonadotrophic activity in the viviparous sea perch Cymatogaster aggregata Gibbons by a dithiocarbamoylhydrazine derivative (ICI 33828). Can. J. Zool. 46, 751-758 - WIEBE, J. P. (1969): Endocrine controls of spermatogenesis and oogenesis in the viviparous sea perch $C y$ matogaster aggregata Gibbons. Gen. Comp. Endrocrinol. 12, 267-275 - WIRTH, W., R. Gosswald, U. Horlein, K. H. Risse und H. Kreiskott (1958): Zur Pharmakologie acylierter Phenothiazin-Derivate. Arch. int. Pharmacodyn. 115, No. 1-2.

Anschrift des Verfassers: Dr. Bernd Kramer, Laboratoire de Physiologie Nerveuse, Groupe des Laboratoires du C.N.R.S., F-91 190 Gif sur Yvette, France. 